\title{
Challenges in assessing the clinical utility and economic value of immune checkpoint inhibitor therapies of Cancer
}

\author{
Peter Paul Yu ${ }^{1 *} \mathbb{D}$, Omar Eton ${ }^{2}$ and Louis P. Garrison ${ }^{3}$
}

\begin{abstract}
Advances in the immunotherapy of cancer have prolonged survival for cancer patients, but the clinical and financial impact of treatments must be considered in determining the overall clinical utility and economic value of therapeutic agents. Quality-adjusted life years and incremental cost-effectiveness ratios are clinical and economic metrics that can be used to evaluate the value of immune checkpoint inhibitors. This Commentary provides perspective on the limitations, benefits, and potential enhancement of this approach to support value-based medicine.
\end{abstract}

Keywords: QALY, Immune checkpoint inhibitors, Cost-effectiveness

The first two decades of the twenty-first Century have yielded truly substantive advances in our understanding of: a) the impact of driver mutations in individual patients' cancers; and b) the dynamic relationship between tumors and the host's ability to mount an effective anti-tumor immune response. In 2005, the number of oncology drugs in clinical development was 359 compared to 586 in 2015, an increase of 63\%. From 2011 to 2015, 70 new cancer drugs were approved but with a significant concomitant increase in healthcare costs [1]. An analysis of all oncology drugs receiving Food and Drug Administration (FDA) approval between 2009 and 2013 found that the median launch price for a course of treatment was $\$ 116$, 100 for drugs with a novel mechanism of action and $\$ 119,765$ for a drug within an established class of agents [2]. Data from the CMS Oncology Care Model for the period January 2014-June 2015 showed that oncologic drugs accounted for $39.1 \%$ of the total cost of care associated with episodes of chemotherapy, a percentage likely to increase and become an increasing threat to economic sustainability [3]. In 2018, the President's Cancer Panel report focused on the rising

\footnotetext{
* Correspondence: Peter.Yu@hhchealth.org

${ }^{1}$ Hartford HealthCare Cancer Institute and Memorial Sloan Kettering Cancer Center, Hartford Hospital, 80 Seymour Street, Hartford, CT 06102-5037, USA Full list of author information is available at the end of the article
}

cost of cancer drugs, their negative impact on patients and the need to relate drug price to the value provided [4].

Value assessment is derived from benefits and costs viewed through clinical, economic, and patient experience domains. Clinical value or utility is the measure of increase in quantity of life (survival) while accounting for impacts on quality of life (QoL) - either decrements due to treatment-related adverse events (AEs) or disease progression, or improvements in functioning and wellbeing due to disease response. The American Society of Clinical Oncology (ASCO) and the European Society for Medical Oncology (ESMO) have both proposed frameworks to assess the clinical utility of cancer therapeutics and these two tools were found to have moderate concordance when evaluated against 97 clinical trials [5]. The value assessments are based on algorithms incorporating survival, quality of life, toxicity and long term survival. Cost is not directly factored into the frameworks, but is considered in three other value frameworks; Memorial Sloan Kettering Drug Abacus [6], National Comprehensive Cancer Network Evidence Blocks [7] and the Institute for Clinical and Economic Review (I.C.E.R.) [8] The value frameworks of both ASCO [9] and I.C.E.R. refer to clinical utility as "net health benefit" (NHB) although they differ in definition. I.C.E.R. employs a more quantitative, event-specific 
model for toxicity assessment as described below. Further, the ASCO value-based framework is constrained to comparisons within a randomized clinical trial and its primary aim is use in shared decision-making between patients and providers, while I.C.E.R. evaluates each treatment's benefit independently but in a manner that enables comparison among different treatments using a healthcare sector and societal perspective.

I.C.E.R defines economic value as the cost to deliver a unit of NHB expressed as the incremental cost-effectiveness ratio (ICER), with net impact on costs (in the numerator) and NHB (in the denominator). These measures can be evaluated a) from different perspectives-societal, health plan, and patient-and b) in different decision contextshealth plan coverage/formulary inclusion, development of clinical guidelines and pathways, and shared clinical decision-making at the patient level [10]. The latter should reflect individual patient's personal preferences and beliefs as well as the out-of-pocket costs they face.

Verma et al. [11] report a systematic review of modeling projections of ICER for immune checkpoint inhibitors (ICI) in four different cancers. The models project the likely ICER over some time horizon based on clinical trial data and mathematical extrapolation of the presumed survival benefit beyond the observed trial data. Furthermore, the models attempt to take into account the quality of life experienced by surviving patients. Thus, the NHB is expressed in terms of the difference in "quality-adjusted life years" (QALY). The QALY measures survival while taking into consideration disease state and AEs. A gain of 1 year in survival is valued at something less than 12 months depending on the impact of disease and AEs on QoL. The ICI models on which these ICER estimates are based are often complex synthetic mathematical models (often Markov health-state transition models) that project overall survival and time spent in disease states of stable disease (SD) and progression of disease (PD), with some models adding time in clinical response. Each disease state is assigned a numeric value called a utility score which is less than 1 (the assumed utility value for perfect health). Immunotherapy-specific criteria of response have been developed (iRECIST) which redefine assignment of disease states [12]. ICI has been associated with longer SD durations than typically seen with chemotherapy, even off therapy, suggesting that some patients with radiographically determined SD may be disease free, and if this is associated with clinical benefit a higher utility value during this prolonged SD may be justified compared to that assigned to chemotherapy associated stable disease [13].

In addition, AEs are assigned negative values called disutilities which are subtracted to arrive at the net QALY. Ideally, the clinical trial on which the ICER is based would have captured the incidence, severity, and duration of AEs utilizing standardized instruments such as the NCI CTCAE v5.0 [14] and QoL assessment tools. Patient-reported outcomes (PRO) instruments (such as the EuroQoL EQ-5D) can be used to estimate health-related quality of life for study patients. A review of PRO measurements reported in case report forms within the FDA database of ICI registration trials for the first 5 ICI found that $75 \%$ of 28 registry trials included at least two PRO instruments. The two most commonly used instruments were EQ-5D in $90 \%$ of trials, and the European Organization for Research and Treatment of Cancer Quality of Life Questionnaire (EORTC QLQ-C30) which was reported in $81 \%$ of the trials. The review identified 8 ICI associated adverse events with frequencies exceeding $20 \%$ and evaluated the capture of these in the PRO instruments used in the registry trials. None of the instruments included AEs of rash or pruritus, and the median number of the 8 adverse events reported was only 3 [15]. Although FDA policy permits the use of PRO in considering regulatory approval, there is no current requirement to include $\mathrm{PRO}$ in registry studies.

The amount that AEs reduce a life-year gained is calculated by applying disutility weights (or "utility decrements") to the trial-based clinical epidemiological parameters of AE incidence, severity, and duration. The studies vary in the utility values attributed to disease states and the disutility values attributed to AEs as seen in Table 1. The values for all studies were obtained from published literature, which are not specific to ICI. Disutility values derived from the literature generally reflect chemotherapy-related AEs. However, some AEs-e.g., pneumonitis or diarrhea-have significantly different clinical impact when a consequence of immunotherapy rather than chemotherapy and disutility values that account for the unique attributes of ICI are not available.

Cost-effectiveness analysis can be applied to comparison of single, combination or sequential use of immunotherapies extending their applicability to cost effectiveness evaluation of immune therapies as new targets and drugs are identified. Kohn et al. used cost-effectiveness analysis to evaluate sequential versus combined ICI in melanoma [16]. The combination of PD-1/PD-L1 and anti-CTLA-4 agents increases both cost and clinical toxicity and sequential therapies starting with a PD-1/PD-L1 were more cost effective than combined therapy with a PD-1/PD-L1 and anti-CTLA-4 agents.

Once a QALY impact has been projected, the cost associated with achieving that QALY gain is expressed in cost-effectiveness analysis as the ICER. Whether that cost provides value depends on the willingness to pay (WTP) from the different perspectives of patients, providers, and public and private payers-but ICER "thresholds" have been most frequently used internationally by government payers working within a prespecified health 
Table 1 Summary of Key Utility Parameter Estimates in Studies Cited in the Systematic Review

\begin{tabular}{|c|c|c|c|c|c|c|}
\hline Author & QoL Source & PF Utility & PD Utility & Pulmonary Disutility & Diarrhea Disutility & Endocrine Disutility \\
\hline Ward & $\begin{array}{l}\text { CheckMate } 141 \text { EQ-5D } \\
\text { QLQ-C30 QLQ-H\&N } 35\end{array}$ & 0.680 & 0.660 & NR & NR & NR \\
\hline Zagar & CheckMate 141 & NR & NR & -0.20010 & -0.20710 & -0.21010 \\
\hline Tringale & CheckMate 141 & 0.517 & 0.280 & NR & NR & NR \\
\hline Goeree & CheckMate 017 EQ-5D & 0.789 & 0.674 & -0.008 & NR & NR \\
\hline Matter Walstra & Literature & 0.756 & 0.470 & NR & NR & NR \\
\hline Aguilar & Literature & 0.65 & 0.43 & NR & NR & NR \\
\hline Huang & KEYNOTE 010 EQ-5D & 0.761 & 0.687 & -0.09 & NR & NR \\
\hline Huang & KEYNOTE 024 EQ-5D & NR & NR & NR & NR & NR \\
\hline Wan & CheckMate 025 EQ-5D & 0.848 & 0.68 & NR & NR & NR \\
\hline McCrea & CheckMate 025 EQ-5D & $\begin{array}{l}\text { Re } 0.895 \\
\text { SD } 0.846\end{array}$ & 0.817 & NR & NR & NR \\
\hline Sarfaty & CheckMate 025 FKSI-DRS & 0.89 & 0.78 & NR & NR & NR \\
\hline Sarfaty & NR & NR & NR & NR & NR & NR \\
\hline Barzey & MDX010-20 SF-36 & $\begin{array}{l}\text { CR } 0.88 \\
\text { SD } 0.80\end{array}$ & 0.52 & NR & NR & NR \\
\hline Curl & Literature and Expert Opinion & $\begin{array}{l}\text { Re } 0.88 \\
\text { SD } 0.80\end{array}$ & 0.52 & NR & -0.09 & NR \\
\hline Bohensky & CA209066 EQ-5D & 0.828 & 0.798 & NR & NR & NR \\
\hline Oh & CheckMate 067 & 0.667 & 0.433 & $-0.159 \mathrm{IO}$ & -0.11610 & $-0.115 \mathrm{IO}$ \\
\hline Wang & KEYNOTE 006 EQ-5D & $0.83-0.86$ & 0.78 & NR & NR & NR \\
\hline Miguel & KEYNOTE 006 EQ-5D & 0.80 & 0.70 & NR & NR & NR \\
\hline Kohn & Literature & $\begin{array}{l}\text { Re } 0.88 \\
\text { SD first line therapy } 0.80\end{array}$ & 0.52 & $-0.17 \mathrm{IO}$ & -0.1710 & -0.1710 \\
\hline Meng & CheckMate 066 EQ-5D & $0.78-0.80$ & $0.71-0.73$ & NR & NR & NR \\
\hline
\end{tabular}

Note: Utility and disutility values used by the systematic review studies

$Q o L$ quality of life, $P F$ progression free, $P D$ progressive disease, $I O$ immune-oncology specific, NR not reported, Re response, $S D$ stable disease, $C R$ complete response

budget. As Medicare is an entitlement program with no fixed budget, there is no federal WTP benchmark in the US. The authors selected a WTP of $\$ 100,000$ as the most widely used figure in the literature, but more recent discussions have argued that a higher number is more appropriate [17]. As the authors note, raising it to $\$ 150,000 /$ QALY would change nivolumab to being costeffective in four of the cancers considered.

The U.S. has no standard or consensus on the appropriate WTP threshold. In its review of about-tolaunch new medicines, I.C.E.R. utilizes a sensitivity analysis of $\$ 50,000$ to $\$ 150,000$ per QALY, but publicizes a "value-based price" based on $\$ 150,000$ per QALY [8]. Their range is roughly 1 to 3 times per capita GDP (Gross Domestic Product), which was $\$ 59,500$ in 2016 [18]. The lower end of range-- $\$ 50$, 000 per QALY - was established in the early 1980s as a benchmark based on the cost of kidney dialysis [19], but it was not adjusted for quality of life nor adjusted over time for inflation or changes in the cost of care. The average annual cost of dialysis for
Medicare in 2016 was $\$ 89,400$. A recent systematic review suggests an average utility for dialysis patients of about 0.6. [20]. The ratio of the two is $\$ 149,000$ $(=\$ 89,400 / 0.6)$, which is at I.C.E.R.'s upper bound. Clearly, every individual has a unique threshold, depending on his or her income, health preferences, and many other factors. Furthermore, different health plans and health systems would have different thresholds, as would different nations. In the United Kingdom (U.K.), the National Institute for Health and Care Excellence (NICE) recently decreased the threshold from GBL30,000 to GBL20,000 per QALY. In 2017, the GDP of the U.K. was GBL30,300 [17].

The cost-per-QALY metric is used most frequently in the decision context of health plan or formulary coverage: i..e., should access to a particular medicine be permitted (but often limited to particular subgroups of patients)? Both the recent Second U.S. Panel on Cost-effectiveness in Health and Medicine [21] and the Special Task Force on U.S. Value Frameworks of the International Society of Pharmaeconomics and Outcomes 
Research (ISPOR) [22] view this question from a "healthcare sector perspective." While health gain in terms of mortality and morbidity improvements are probably what matter most to patients, both reports cite other elements that should be considered in a broader "societal perspective", such as impacts on productivity, family members and caregivers, scientific knowledge spillovers, and uncertainty related to financial risk protection and the likelihood of benefit, among others.

While the above discussion considers cost-effectiveness of ICI for broad populations, subgroups of patients defined by clinical features may benefit to greater or lesser degree. Verma et al. point out that more focused selection of patients for treatment using host and tumor-related biomarkers could improve the effectiveness of ICI, and thereby the QALY gained, by identifying sub-populations with higher likelihood of receiving benefit or reduced toxicity. The FDA requires biomarker testing in some cancers (companion diagnostic) and recommends testing for others (complementary diagnostic). To the degree that biomarkers identify patients who are more likely to respond to ICI and lead to better clinical choices among treatment options they will increase the QALY gain of the drug. Four biomarkers are currently used to predict degrees of immunotherapy responsiveness but are not generally measured together: microsatellite instability (MSI), tumor mutation burden (TMB), PD-L1 expression and immune cell infiltrate in or around the tumor. MSI has achieved FDA approval as a biomarker that permits selection of immunotherapy with a high probability of achieving clinical benefit; however, such positivity is a relatively uncommon occurrence. MSI is a surrogate marker for deficiencies in DNA repair and similar to TMB is a surrogate marker for increased tumor related antigenicity as tumor mutations lead to neo-antigens that are potential targets of the immune system [23]. With the increased use of next-generation sequencing, TMB can be calculated based on the percentage of nonsynonymous somatic mutations per megabase sequenced and is being used to report patient TMB [24]. Yet although high TMB may increase the likelihood that an endogenous host immune response has been generated but suppressed by checkpoint mechanisms, it does not ensure that. Further, the nature of the cancer antigen in terms of protein function and immunogenicity may be more important than the number of antigen targets on the cancer cell. PD-L1 expression by immunohistochemistry can be a surrogate marker for suppression of a host immune response to the cancer and is being used to select patients for treatment for some cancers. However, the variability in testing platforms and cut off levels used to predict response, and the inconsistent predictive value of the test in different cancers has limited the utility of PD-L1 testing as recently reviewed [25]. The presence of immune cells bearing PD-L1 either surrounding or infiltrating the cancer has been suggested to predict ICI responsiveness by identifying what are referred to as hot cancers, although quantitative assessment of the degree of $\mathrm{T}$ cell infiltration and qualitative assessment of patterns of host immune response using immune cell biomarkers is likely to be beyond unaided human cognitive capacity Advances in the use of image recognition using artificial intelligence combined with multiple cell surface markers that can identify immune effector cell populations would enhance our understanding of the orchestration of the host immune response. A similar strategy can be pursued using gene expression analysis to predict downstream activation of immune activation pathways as has been reported for interferon gamma, a key positive and negative modulator of the immune system [26]. Greater precision in biomarkers keyed to specific aspects of regulation of the immune response and tailored to both the patient and cancer type will improve the clinical value of both ICI and emerging classes of immunotherapy of cancer.

How can we move forward an augmented QALYbased analysis to implement a more rational coverage policy that ensures access to the most advanced oncologic therapies while not simply paying whatever the market will endure irrespective of actual NHB gained? We suggest seven considerations.

First, it is generally unlikely-and certainly unclearwhether the drug prices used in these projections reflect the cost actually paid since confidential discounts are a common feature of our drug reimbursement system [17]. Greater transparency on how price is escalated as drugs move through the supply chain and specialty pharmacy distributors is needed.

Second, these are mathematical projection models based on clinical trial data often for regulatory approval: this is the best information available at launch, but it may be poor predictor of future real-world application and outcomes. This is an argument for a healthcare system that gathers post-launch real-world evidence and adjusts prices (i.e., the rewards to manufacturers) based on the actual value delivered [27].

Third, disutility values specific to immune-oncology need to be estimated through the use of PRO instruments germane to immunotherapy related adverse events. This will require either the design of new PRO instruments or modification of existing instruments currently used in oncology studies.

Fourth, value each incremental gain in longevity equally using the QALY although prolonged survival (tail of the survival curve), especially if off-treatment, might merit extra weight as is done in the ASCO and ESMO models. This approach stems from the observation that patients who have responded to ICI can have 
prolonged survival even off therapy suggesting that some of these patients may be cured.

Fifth, although the mean introduction price of a new ICI drug often exceeds $\$ 100,000$, it is worth remembering that the marginal cost of making and distributing the medicine is far below this. Hence, we are really arguing about how much of a reward to give to the manufacturer for this innovation for a particular indication, but doing so based on very limited information at launch. The ICER value for an ICI will vary according the effectiveness of the agent in different cancer types. A good case can be made for "indication-specific pricing" (moving away from per-tablet or per-vial reimbursement) since the ICER will vary by indication (by cancer or line of therapy) at a constant price per tablet or vial [28]. Efforts are underway to implement such programs, perhaps through differential confidential discounts in manufacturer-payer contracts.

Sixth, ICERs will have limited uptake in the U.S. if their use does not acknowledge the high value our nation's culture invests in respecting the patients' autonomy and the physician-patient relationship embedded in shared-decision making. More specifically, utility and disutility values applied to QALY need to be refined based on PRO and clinician input. If the inputs into a QALY-based value assessment are viewed as arbitrary or dependent upon one-size-fits all determinants, we will not build the political will for change.

Seventh, oncologists must take a lead role in delineating transparent consensus and evidence-based treatment pathways. Consideration of cost-effectiveness, and thus ICERs, should be a foundational part of that evidence base.

The rapid progress in unraveling the complexity of host immune response to cancer and its constituent components such as T-regulatory cells, myeloid cells, cytokines and metabolic products of the human microbiome bodes well for patients with cancer and the physicians who study and treat these diseases. At the same time the impact of these clinical advances on the health system sustainability threatens equitable access for patients to life sustaining-treatments. Developing predictive biomarkers that identify which classes of immune-oncologic agents such as ICI provide the most benefit to a given patient will be essential and should be part of drug development. At the same time, accurately capturing adverse events of therapies using PRO will inform the overall value of a new therapeutic agent. As we create new classes of immune-oncology agents, combination or sequential therapies will be used, and teasing out the contribution of each agent to the overall outcomes of the patient will be difficult given current health economic measurement tools in use. It is not likely that assessing the value of therapy, accounting for its clinical and financial benefit and cost on both patient and societal levels, will be feasible without real-world, post drug approval evidence, which will necessitate capturing patient treatment care data outside the context of a clinical trial and require joint efforts on the part of professional societies, payers, pharmaceutical industry and government. Data will need to be collected during routine clinical care delivery and as part of quality improvement efforts and health economic research efforts using instruments such as PRO and electronic health records calibrated to collect such data without the need for extensive manual extraction and clean up as is now the norm. Payers, government agencies and industry have a critical role funding evidence collection through performance-based risk sharing arrangements [29]. Value frameworks will have limited utility unless they are able to incorporate such real-world evidence. Value frameworks are easily criticized for their imperfections, difficult to understand methodology and inability to address the perspectives of multiple stakeholders. Yet it is important to understand that these imperfect models provide the basis for rational discourse among patients, healthcare providers and systems, industry, payers and governments.

\section{Abbreviations}

AEs: Adverse events; GDP: Gross domestic product; I.C.E.R.: Institute for clinical and economic review; ICER: Incremental cost-effectiveness ratio; ICI: Immune checkpoint inhibitors; MSI: Microsatellite instability; NHB: Net health benefit; PD: Progression of disease; PRO: Patient-reported outcomes; QALYs: Quality-adjusted life years; QoL: Quality of life; SD: Stable disease; TMB: Tumor mutation burden; WTP: Willingness-To-Pay

\section{Acknowledgements}

Not Applicable

\section{Authors' contributions}

PPY abstracted data for Table 1 and was a major contributor in writing the manuscript, OE was a major contributor in writing the mauscript, LPG was a major contributor in writing the manucript. All authors read and approved the final manuscript.

\section{Funding}

Not Applicable

\section{Availability of data and materials Not Applicable}

Ethics approval and consent to participate Not Applicable

\section{Consent for publication \\ Not Applicable}

\section{Competing interests}

Peter Paul Yu: no competing interests, Omar Eton: Dr. Eton participates in the Merck speakers bureau and is an investigator on a BMS clinical trial, Louis P. Garrison: Professor Garrison has provided economic consultation and participated in advisory boards for several companies with an interest in 10 products, including BMS, Pfizer, Roche, MSD and Novartis. He is currently a member of the ASCO Methodology Working Group for Value in Cancer Care Task Force.

\section{Author details}

${ }^{1}$ Hartford HealthCare Cancer Institute and Memorial Sloan Kettering Cancer Center, Hartford Hospital, 80 Seymour Street, Hartford, CT 06102-5037, USA. 
${ }^{2}$ Hartford HealthCare Cancer Institute, 80 Seymour Street, Hartford, CT 06102-5037, USA. ${ }^{3}$ The Comparative Health Outcomes, Policy, and Economics (CHOICE) Institute, University of Washington, Health Sciences Building, H375, 1959 NE Pacific Street, Box 357630, Seattle, WA 98195-7630, USA.

\section{Received: 20 January 2019 Accepted: 14 August 2019}

Published online: 03 September 2019

\section{References}

1. IMS Institute for Healthcare Informatics. Global oncology trend report: a review of 2015 and outlook to 2020. Parsippany: IMS Institute for healthcare informatics; 2016. Accessed 23 June 2019. Available from: https:/www.iqvia.com/-/media/iqvia/ pdfs/institute-reports/global-oncology-trend-report-2016.pdf

2. Mailankody S, Prasad V. Five years of cancer drug approvals: innovation, efficacy, and costs. JAMA Oncol. 2015;1(4):539-40 Accessed 7 Jan 2019. Available from: https://www.ncbi.nlm.nih.gov/pubmed/26181265.

3. First Annual Report From the Evaluation of the Oncology Care Model: Baseline Period. Accessed 2 Jan 2019. https://downloads.cms.gov/files/ cmmi/ocm-baselinereport.pdf

4. Promoting Value, Affordability, and Innovation in Cancer Drug Treatment. https://prescancerpanel.cancer.gov/report/drugvalue/ Accessed 31 Dec 2018.

5. Cherny NI, de Vries EGE, Dafni U, al e. Comparative assessment of clinical benefit using the ESMO-magnitude of clinical benefit scale version 1.1 and the ASCO value framework net health benefit score. J Clin Oncol. 2018;37: 336-49. https://doi.org/10.1200/JCO.18.00729.

6. Drug Pricing Lab Memorial Sloan Kettering. https://drugpricinglab.org/tools/ drug-abacus/ Accessed 23 June 2019.

7. NCCN Clinical Practice Guidelines in Oncology (NCCN Guidelines) with NCCN Evidence Blocks. https://www.nccn.org/evidenceblcoks/ Accessed 23 June 2019.

8. Institute for Clinical and Economic Review. Overview of the ICER value assessment framework and update for 2017-2019, January 2018. Available at: https://icer-review.org/wp-content/uploads/2018/03/ICER-valueassessment-framework-update-FINAL-062217.pdf. Accessed 8 Jan 2019.

9. Schnipper L, Davidson N, Wollins DS, Blayney DW, Dicker AP, Ganz PA, Hoverman JR, Langdon RM, Lyman GH, Meropol NJ, Mulvey TM, Newcomer L, Peppercorn J, Polite B, Raghavan D, Rossi GP, Saltz LB, Schrag D, Smith TJ, Yu PP, Hudis CA, Vose JM, Schilsky RL. Updating ASCO's value framework: revisions and reflections in response to comments received. J Clin Oncol. 2016:34:2925-34. https://doi.org/10.1200/JCO.2016.68.2518.

10. Garrison LP Jr, Pauly MV, et al. An overview of value, perspective, and decision context-a health economics approach: an ISPOR special task force report [2]. Value Health. 2018;21(2):124-30.

11. Verma V, Spave T, Haque W, Simone CB, Chang JY, Welsh JW, Thomas CR. A systematic review of the cost and cost-effectiveness studies of immune checkpoint inhibitors. J Immunother Cancer. 2018;6:128. https://doi.org/ 0.1186/s40425-018-0442-7.

12. Seymour L, Bogaerts J, Perrone A, al e. iRECSIT: guidelines for response criteria for use in trials testing immunotherapeutics. Lancet Oncol. 2017; 18(3):e143-52. https://doi.org/10.1016/S1470-2045(17)30074-8.

13. Gulley JL, Rajan A, Spigel DR, et al. Avelumab for patients with previously treated metastatic or recurrent non-small-cell lung cancer (JAVELIN solid tumor): dose-expansion cohort of a multicenter, open-label, phase 1b trial. Lancet Oncol. 2017;18(5):599-610.

14. National Cancer Institute. Common terminology criteria for adverse events (CTCAE). Available at : https://ctep.cancer.gov/protocoldevelopment/ electronic_applications/ctc.htm. Accessed 8 Jan 2019.

15. King-Kallimanis BL, Howie $L$, Roydhouse JK. Et.al. Patient reported outcomes in anti-PD-1/PD-L1 inhibitor immunotherapy registration trials: FDA analysis of data submitted and future directions. Clin Trials. 2019;16(3):322-6. https:// doi.org/10.1177/1740774519836991

16. Kohn CG, Zeichner SB, Chen Q, et al. Cost-effectiveness of immune checkpoint inhibition in BRAF wild-type advanced melanoma. J Clin Oncol. 35:1194-202. https://doi.org/10.1200/JCO.2016.69.6336.

17. Garrison LP, Jackson T, Paul D, Kenston M. Value-based pricing for emerging gene therapies : the economic case for a higher cost-effectiveness threshold. J Manag Care Spec Pharm. 2019;25(7):793-9.

18. The World Bank. (current international PPP\$), World development indicators database, World Bank. Database updated on 1 July 2017. Available at:
https://data.worldbank.org/indicator/NY.GDP.PCAP.CD?end=2017\&start= 2016. Accessed 8 Jan 2019.

19. Grosse SD. Assessing cost-effectiveness in healthcare: history of the $\$ 50,000$ per QALY threshold. Expert Rev Pharmacoecon Outcomes Res. 2008;8(2):165-78.

20. Wyld M, Morton RL, Hayen A, et al. A systematic review and meta-analysis of utility-based quality of life in chronic kidney disease treatments. PLoS Med. 2012;9(9):e1001307.

21. Neumann PJ, Sanders GD, Russell LB, Siegel JE, Ganiats TG. Cost-effectiveness in health and medicine. New York: Oxford University Press; 2016.

22. Garrison LP, Neumann PJ, Willke RJ, et al. A health economics approach to US value assessment frameworks - summary and recommendations of the ISPOR special task force report [7]. Value Health. 2018;21(2):161-5.

23. Chalmers ZR, Connelly CF, Fabrizio D, et al. Analysis of 100,000 human cancer genomes reveals the landscape of tumor mutation burden. Genome Med. 2017;19:34. https://doi.org/10.1186/s13073-017-0424-2.

24. Samstein RM, Lee $\mathrm{CH}$, Shoushtari $\mathrm{AN}$, et al. Tumor mutational load predicts survival after immunotherapy across multiple cancer types. Nat Genet. 2019; 51:202-6. https://doi.org/10.1038/s41588-018-03312-8.

25. Signorelli D, Giannatempo P, Grazia G. Patients selected for immunotherapy in solid tumors: overcome the naïve vision of a single biomarker. Biomed Res Int. 2019. https://doi.org/10.1155/2019/9056417.

26. Ayers M, Lunceford J, Nebozhyn M. IFN-gamma-realted nRNA profile predicts clinical response to PD-1 blockade. J Clin Invest. 2017;127(8):293040. https://doi.org/10.1172/JCl91190.

27. Garrison LP, Jr. (co-chair); Towse a (co-chair) et al. Performance-based risksharing arrangements - good practices for design, implementation, and evaluation: report of the ISPOR good practices for performance-based risksharing arrangements task force. Value in Health. 2013.

28. Pearson SD, Dreitlein WB, et al. Indication-specific pricing of pharmaceuticals in the US healthcare system. J Comp Eff Res. 2017:6(5):397-404.

29. Garrison LP, Towse A, Briggs A, al e. Performance-based risk-sharing arranagements-good practices for design, implementation, and evaluation: report of the ISPOR good practices for performance-based risk-sharing arrangements task force. Value Health. 2013;16:703-19. https://doi.org/10.1 016/j.val.2013.04.011

\section{Publisher's Note}

Springer Nature remains neutral with regard to jurisdictional claims in published maps and institutional affiliations.

Ready to submit your research? Choose BMC and benefit from:

- fast, convenient online submission

- thorough peer review by experienced researchers in your field

- rapid publication on acceptance

- support for research data, including large and complex data types

- gold Open Access which fosters wider collaboration and increased citations

- maximum visibility for your research: over $100 \mathrm{M}$ website views per year

At BMC, research is always in progress.

Learn more biomedcentral.com/submission 